\title{
Perfil da Dispensação de Medicamentos Controlados pelo Sistema Único de Saúde de Boa Vista do Incra - RS
}

\author{
Profile of the Dispensing of Controlled Drugs by the Unified Health \\ System of Boa Vista do Incra - RS
}

\section{RESUMO}

Objetivos: Avaliar quais os medicamentos controlados foram dispensados com maior frequência no Município de Boa Vista do Incra - RS. Metodologia: Trata-se de um estudo descritivo de caráter quantitativo de análise documental do banco de dados de dispensação da farmácia do município, nos meses de agosto e setembro de 2019. Resultados: Ao analisar as prescrições do referido período, verificou-se maior frequência de prescrição de quatro medicamentos: alprazolam $(12,7 \%)$, citalopram $(12,4 \%)$, amitripilina $(13,7 \%)$ e sertralina $(7,4 \%)$. Os dados encontrados demonstram maior frequência de prescrição de medicamentos psicoativos, corroborando dados de estudos acerca do assunto. Conclusões: Os dados revelaram uma elevada dispensação de medicamentos controlados, tendo em vista o número de habitantes no município estudado. Esses medicamentos apresentam a capacidade de provocar reações adversas, destacando-se a importância da atuação do profissional farmacêutico na promoção do uso racional de medicamentos, de forma a reduzir os riscos causados por essa classe medicamentosa.

\section{DESCRITORES}

Prescrição de Medicamentos. Relação Profissional Paciente. Sistema Único e Saúde.

\begin{abstract}
Objective: The objective of this study was to evaluate which controlled dispensing drugs were dispensed more frequently in the city of Boa Vista do Incra - RS. Methodology: This is a descriptive study of quantitative approach, and a documentary analysis of the dispensing database of the pharmacy of the city in the months of August and September 2019. Results: When analyzing the prescriptions of the months of August and September 2019, higher frequency of prescription of four drugs: alprazolam (12.7\%), citalopram (12.4\%), amitripillin (13.7\%) and sertraline $(7.4 \%)$ were found. The data shows higher frequency of prescription of psychoactive drugs corroborating with data from studies on the subject. Conclusion: The data reveal a high dispensation of controlled drugs, given the number of inhabitants studied in the city. These drugs have the ability to cause adverse reactions, highlighting the importance of the role of the pharmaceutical professional in promoting the rational use of drugs, in order to reduce the risks caused by this drug class.
\end{abstract}

\section{DESCRIPTORS}

Drug Prescriptions. Professional Patient Relations. Unified Health System.

${ }^{1}$ Farmacêutica, Universidade de Cruz Alta, Centro de Ciências da Saúde e Agrárias, Cruz Alta, RS, Brasil.

${ }^{2}$ Farmacêutica, Universidade de Cruz Alta, Mestranda do Programa de Pós-Graduação em Atenção Integral a Saúde, Cruz Alta, RS, Brasil.

${ }^{3}$ Doutor em Química, Farmacêutico, Centro Universitário Metodista - IPA, Cruz Alta, RS, Brasil.

${ }^{4}$ Doutora em Biologia Celular e Molecular, Farmacêutica, Universidade de Cruz Alta, Centro de Ciências da Saúde e Agrárias, Cruz Alta, RS, Brasil.

${ }^{5}$ Doutora em Farmacologia, Farmacêutica, Universidade de Cruz Alta, Programa de Pós-Graduação em Atenção Integral a Saúde, Cruz Alta, RS, Brasil.

${ }^{6}$ Doutora em Ciências Farmacêuticas, Farmacêutica, Universidade de Cruz Alta, Centro de Ciências da Saúde e Agrárias, Cruz Alta, RS, Brasil. 
$\mathrm{O}$ s medicamentos controlados são dispensados para uso em humanos de forma preconizada por meio da prescrição médica ou odontológica, sendo tal prática normatizada pela Portaria $\mathrm{N}^{\circ}$. 344, de 12 de maio de 1998. A iniciativa desta normativa parte do uso irracional e indevido de grupos de medicamentos capazes de gerar agressões ao organismo, bem como, desencadear quadros de dependência e de agravamento de determinadas condições patológicas ${ }^{1}$.

Dentre os medicamentos controlados se observa uma classificação de acordo com suas características, sendo esta classificação separada em medicamentos do grupo A, B e $C$ e seus subgrupos. Além disso, existe uma classificação estabelecida pela portaria que categoriza os medicamentos como sendo: entorpecentes (A1 e A2), psicotrópicos (A3, B1 e B2) ou outras substâncias sujeitas a controle especial $(\mathrm{C} 1)^{2}$.

Fazem parte dos medicamentos controlados, os psicotrópicos, pertencentes ao grupo B, mais especificadamente, os subgrupos B1 psicotrópicos e B2, psicotrópicos anorexígenos. Nos últimos anos, observou-se um rápido crescimento no uso de psicotrópicos, não apenas no Brasil, mas no mundo todo. Desta forma, desencadeia-se um estado de alerta devido ao potencial que estes fármacos possuem em gerar quadros de dependência química ${ }^{3}$.

Devido ao cenário de preocupações em se realizar o uso racional dos medicamentos controlados, especialmente os psicotrópicos e, desta forma reduzindo o abuso destas substâncias, a Organização Mundial de Saúde (OMS) desenvolveu indicadores de prescrição, de forma a avaliar a prática farmacêutica de dispensação e de liberação de medicamentos em centros de saúde de pequeno porte . $^{4}$

Com base nos dados obtidos por meio destes indicadores de prescrição e consumo de medicamentos, é possível realizar uma avaliação situacional e, desta forma, o desenvolvimento de estratégias que auxiliam na redução do uso destes fármacos, modulando a distribuição de acordo com as necessidades populacionais ${ }^{5}$.

Diante deste contexto, este estudo teve como objetivo, avaliar o perfil de dispensação de medicamentos controlados em uma farmácia de um município de pequeno porte, com base nas dispensações efetuadas pela farmácia municipal.

\section{METODOLOGIA}

Trata-se de uma pesquisa descritiva, quantitativa com análise documental de forma transversal, que buscou avaliar quais os medicamentos controlados foram prescritos com maior frequência na farmácia municipal de Boa Vista do Incra, no interior do Rio Grande do Sul, no período de agosto a setembro de 2019.

O presente estudo foi aprovado pelo Comitê de Ética em Pesquisa sob o parecer consubstanciado $n^{\circ}$. 3.165.283.

Os dados foram coletados da base de dados do Sistema informatizado de gestão em saúde (TEÍ Saúde), com auxílio de um mapa de coleta de dados contemplando as variáveis: o sexo, os medicamentos prescritos e o tipo de medicamento.

Os dados foram analisados utilizando-se o software Microsoft Office 2013 - Excel. 
Para a análise das variáveis categóricas foi utilizada frequência relativa e absoluta e para as variáveis quantitativas, a média e o desvio padrão (DP).

\section{RESULTADOS}

Boa Vista do Incra é um município do interior do Rio Grande do Sul, Brasil, localizado no noroeste do estado, com uma população predominantemente de imigrantes alemães e a economia baseada na agricultura, na pecuária e nas indústrias. A população estimada em 2010 era de 2.425 habitantes $^{6}$. A rede pública de saúde conta com uma Unidade Básica de Saúde (UBS) e uma Equipe de Estratégia de Saúde da Família (ESF) com 100\% de cobertura e com equipes do programa de agentes comunitários de saúde ${ }^{7}$, sendo que a pesquisa foi realizada com os dados obtidos na UBS. Um fator limitante do sistema foi o fato de que não se tem registro das idades dos pacientes e, desta forma, não foi possível a obtenção destes dados.

Foi observado um total de 480 dispensações entre os mais variados tipos de medicamentos controlados pela Portaria $\mathrm{n}^{\circ}$. 344, no período de estudo. Com isso, levando-se em conta a população local, observou-se que $19,5 \%$ da população fez uso de medicamentos controlados. Em relação a distribuição por sexo, predominou as dispensações para o sexo feminino $(n=248,52 \%)$.

Os medicamentos mais prescritos nos meses de agosto e setembro de 2019, estão ilustrados nas Figuras 1 e 2, respectivamente. Em relação as prescrições avaliadas no mês de agosto, verificou-se um total de 231 medicamentos controlados dispensados, dos quais foi observada uma maior frequência de dispensação de três medicamentos: Alprazolam $(n=42,18,2 \%)$, Citalopram $(n=35$, $15,5 \%$ ) e Amitripilina ( $n=39,16,9 \%)$. No mês de setembro, foram dispensados 311 medica-

Figura 1. Frequência relativa de medicamentos prescritos em agosto de 2019

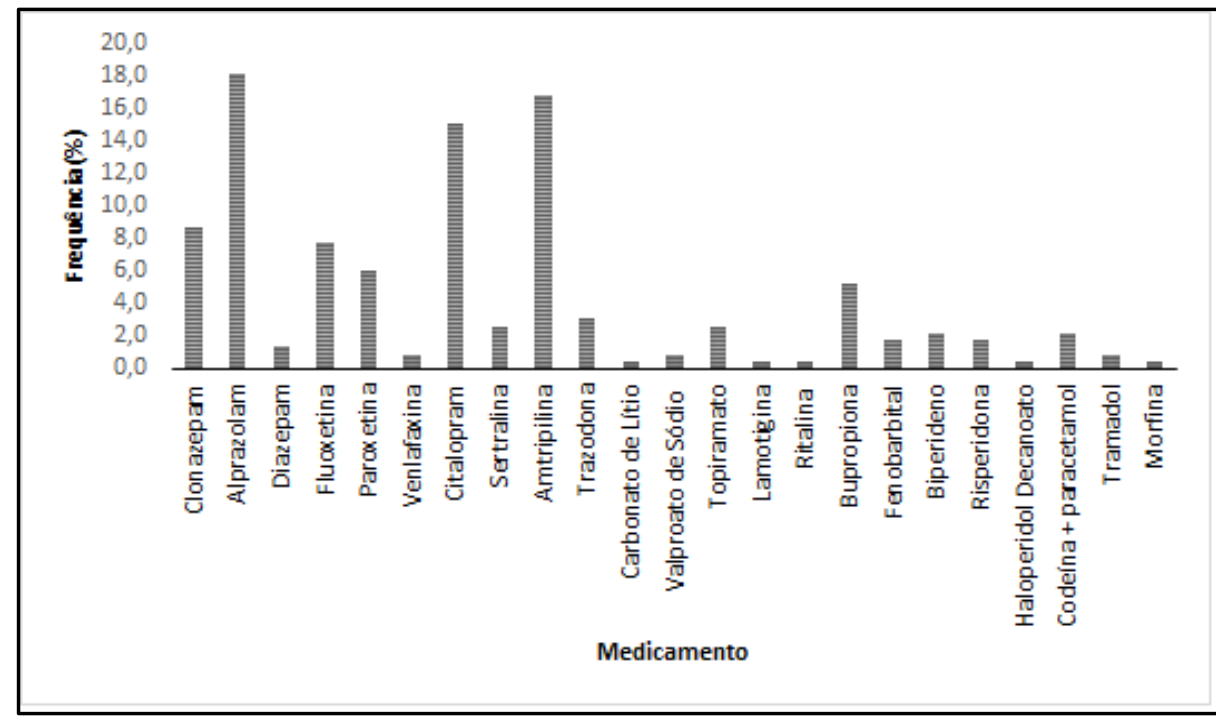


mentos de prescrição controlada, sendo 4 os mais frequentes: Alprazolam $(n=52,16,7 \%)$, Citalopram $(n=30,9,6 \%)$, Sertralina $(n=38$, $12,2 \%$ ) e Amitripilina ( $n=72,10,6 \%)$.

Quando analisada a média das prescrições dos dois meses foram observados maiores frequenciais de prescrição de quatro medicamentos, Alprazolam (12,7\%), Citalo- pram (12,4\%), Amitriptilina(13,7) e Sertralina $(7,4 \%)$ (Figura 3).

\section{DISCUSSÃO}

O emprego do termo dispensação foi incialmente conhecido de forma legal em 1973, sendo utilizado para indicar o forneci-

Figura 2. Frequência relativa de medicamentos prescritos em setembro de 2019

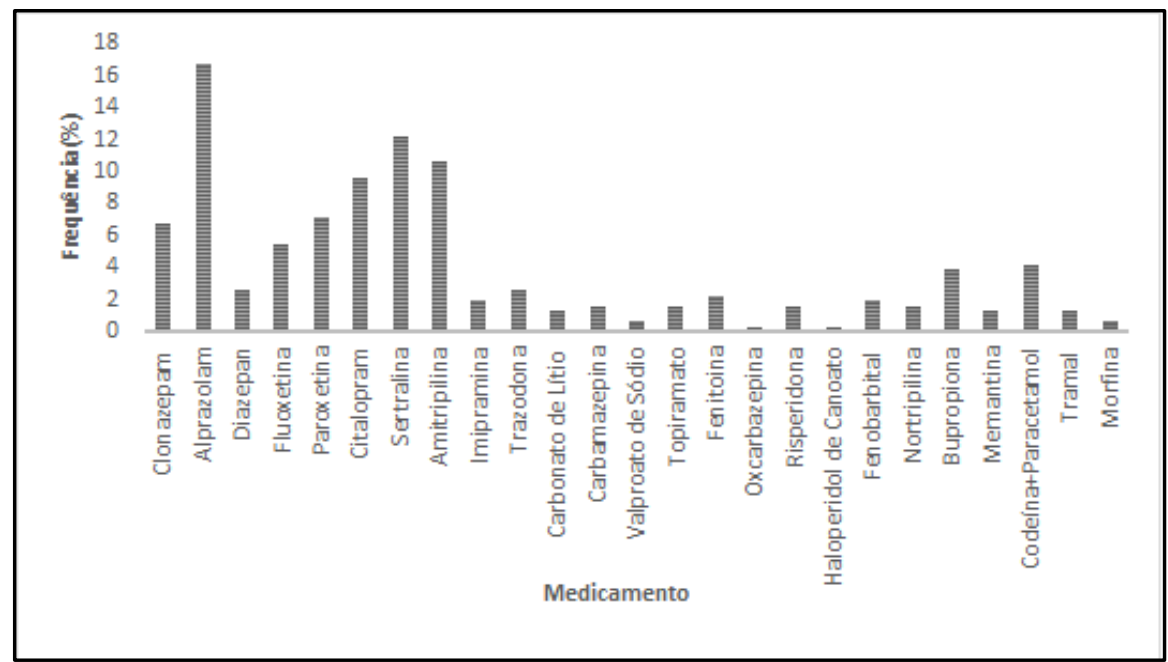

Figura 3. Frequência relativa total de medicamentos mais prescritos nos meses de agosto e setembro de 2019

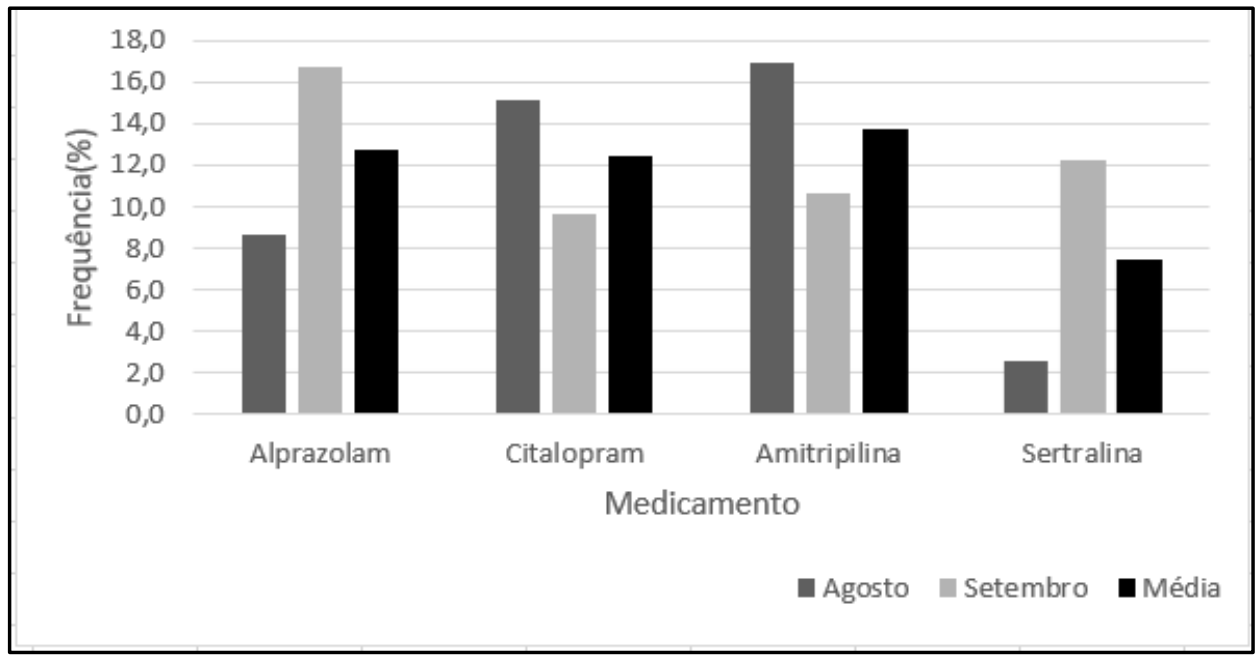


mento de medicamentos aos clientes consumidores com ou sem prescrição. De uma maneira geral, mundialmente, a dispensação de medicamentos vem sendo negligenciada de certa forma, em que o ato se limita a simples entrega do medicamento sem que haja um momento útil de orientações feitas pelos profissionais farmacêuticos, se tornando assim algo simplista ${ }^{8}$. Por este motivo, foi criada a Lei $n^{\circ} 13.021$ de 2014, com o objetivo de trazer à tona a farmácia como sendo um estabelecimento de saúde, ou seja, um ambiente onde a assistência farmacêutica deve ser prestada $^{9}$, reforçando o que está presente na Lei $\mathrm{n}^{\circ}$. 338 de 2004, que trata da Política Nacional de Assistência farmacêutica ${ }^{10}$.

No sistema único de saúde (SUS), uma série de esforços vem sendo realizados com o intuito de melhorar e qualificar os serviços realizados pelos farmacêuticos e incentivar o uso racional de medicamentos ${ }^{8}$. Uma das ferramentas desenvolvidas pela Agência Nacional de Vigilância Sanitária (ANVISA), para as vigilâncias sanitárias locais e o controle de dispensação de medicamentos de controle especial, foi o Sistema Nacional de Gerenciamento de Produtos Controlados (SNGPC), descrito na RCD n. 22 de 2014. Essa resolução estabelece que todas as drogarias e farmácias devem registrar os medicamentos dispensados, inclusos nas listas da Portaria $n^{\circ} .344$ de 1998, no SNGPC ${ }^{2,11}$.

Em relação a distribuição das prescrições por sexo, foi possível observar uma discreta prevalência no sexo feminino, representando $52 \%$ do total de prescrições. Esse dado pode estar relacionado com o fato de a população brasileira ser composta na maioria por mulheres, representando $51,7 \%$ em rela- ção aos homens, de acordo com o IBGE ${ }^{12}$. Em um estudo realizado por Santin e Roman-Júnior ${ }^{13}$, em que avaliaram a dispensação de medicamentos pelo sistema único de saúde (SUS) nas farmácias básicas do município de Vargem Bonita-SC, também ocorreu predomínio do sexo feminino, correspondendo a $69 \%$ dos participantes da pesquisa.

O uso em larga escala de alguns fármacos psicoativos se tornou algo cada vez mais presente na sociedade humana e, isso, se deve principalmente ao aumento do estresse, causado pelos mais diversos fatores, como o trabalho, a escola, os compromissos, entre outros, que podem influenciar negativamente na saúde mental do indivíduo, levando o mesmo ao adoecimento. Pesquisas com caráter epidemiológico no Brasil demonstraram que, entre os medicamentos consumidos com maior frequência pela população estão os antidepressivos e os ansiolíticos, com ênfase nos benzodiazepínicos, que superam os demais na frequência de uso ${ }^{14}$.

Os fármacos antidepressivos fazem parte de um grupo de medicamentos utilizados no tratamento de desordens como os quadros patológicos de depressão. Estes medicamentos atuam no sistema nervoso central em portadores de algum desequilíbrio bioquímico de neurotransmissores, principalmente, de forma que não influenciam significativamente no organismo em seu estado normal ${ }^{15}$.

Em nosso estudo, foi possível observar que o medicamento prescrito com maior frequência foi o alprazolam, um medicamento pertencente a classe dos benzodiazepínicos e utilizado no tratamento de desordens psiquiátricas como síndrome do pânico e ansiedade generalizada. Contudo, em relação à prática 
clínica, existem certas discrepâncias nas opiniões dos especialistas, devido ao fato que a maioria considera o medicamento como sendo altamente viciante, devido as suas propriedades psicodinâmicas relacionadas ao uso. Entretanto, a grande parcela dos clínicos da atenção básica realizam prescrições por longos períodos de tempo, mesmo após ter superado o período estipulado para o tratamento $^{16}$.

O uso do alprazolam em larga escala e, em alguns casos de maneira abusiva, já é retratado na literatura há algum tempo e alguns estudos demonstram o seu uso exacerbado $^{17,18}$. Com isso, os achados deste estudo corroboram os índices de prescrição deste medicamento pelos profissionais, o que gera um estado de atenção para o seu uso excessivo que apresenta potencial de gerar danos ao organismo, quando utilizado de forma inadequada ${ }^{19}$.

No estudo realizado por Silva et al. ${ }^{20}$, em uma farmácia comunitária de São Luiz Gonzaga-RS, foi demonstrada a prevalência de $8,6 \%$ na dispensação de alprazolam, um pouco diferente dos resultados obtidos neste estudo que demostrou uma média de $17,45 \%$.

O citalopram, o segundo mais dispensado entre os meses de agosto e setembro, é um fármaco pertencente à classe dos inibidores seletivos da recaptação de serotonina (ISRS), utilizado principalmente no tratamento da depressão ${ }^{21}$. Os medicamentos da classe dos ISRSs, em apenas uma década, passaram a ser mais utilizados do que os antidepressivos tricíclicos (ADTS).

O motivo pelo qual os ISRSs são populares se deve ao fato que apresentam menores efeitos colaterais em relação aos demais medicamentos utilizados para este fim. Além disso, os pacientes prezam por não ter efeitos secundários negativos ao tratamento, o que garante uma maior adesão. Outros aspectos relacionados ao uso em larga escala também se deve ao fato de que esses medicamentos atingem mais facilmente uma dosagem terapêutica, além de terem um largo espectro no tratamento de uma ampla quantidade de psicopatologias ${ }^{22}$.

No entanto, sendo um fármaco que atua no sistema nervoso central (SNC), o seu uso deve ser acompanhado e realizado com cautela, devido aos riscos relacionados a hiponatremia associada ao uso do citalopram ${ }^{23}$.

Números significativos de prescrições de citalopram são uma realidade no cenário de atenção básica no Rio Grande do Sul. Um estudo realizado por Schenkel e Colet $^{24}$, em São José do Inhacorá/RS, em que avaliaram a frequência de medicamentos antidepressivos dispensados no município, demonstrou que a frequência de dispensação do citalopram foi de $18,1 \%$, corroborando os resultados desse estudo, em que a frequência foi de 12,4\%, em relação aos demais medicamentos. Todavia, existem estudos apontando o aumento do intervalo QT corrigido (QTC), que representa a duração da sístole elétrica, corrigia de acordo com a frequência cardíaca, com o uso do citalopram de maneira errônea, desta forma reforçando os cuidados que se deve ter no uso deste medicamento ${ }^{25}$.

A amitriptilina pertence à classe dos antidepressivos tricíclicos (ADT) sendo que sua ação farmacológica se baseia no aumento da concentração de serotonina na fenda sináptica, contribuindo para o aumento de 
norepinefrina no SNC, pela ação inibitória de recepção deste neurotransmissor ${ }^{26}$.

Este medicamento é utilizado para o tratamento de depressão devido aos efeitos que exerce no individuo ${ }^{27}$. Neste estudo, a amitriptilina esteve entre os medicamentos mais prescritos e se tem relato na literatura de grande número de prescrições e usos deste antidepressivo, mostrando, com isso, que a realidade de prescrição observada nesta avaliação, encontra-se dentro do que se observa em outras regiões ${ }^{28}$.

O presente estudo demonstrou uma média das frequências dos meses de agosto e setembro de $13,8 \%$ de prescrição de amitripilina, um dado semelhante a este foi encontrado no estudo de Gonçalves et al. ${ }^{29}$, em que realizaram a avaliação das prescrições de fármacos psicoativos e evidenciaram uma frequência de 18,1.

A sertralina faz parte dos medicamentos utilizados no tratamento da depressão, ansiedade, síndrome obsessiva compulsiva, entre outras desordens psíquicas. No entanto, esse medicamento não é isento de reações adversas, que estão relacionadas a efeitos serotoninérgicos, como a insônia, a diarreia, o vômito, a cefaleia, a ansiedade, a agitação, o tremor e a disfunção sexual. Com isso, o uso irracional pode ser prejudicial ao organismo ${ }^{22}$.

Em um estudo realizado por Alves e Oliveira $^{30}$, em relação a dispensação de antidepressivos, foi observado uma frequência $3 \%$ para a sertralina no SUS. No entanto, no

\section{REFERÊNCIAS}

1. Mota IVR, Nascimento LES, Cardoso RA, Lemos LB, Lemos GS. Medicamentos sob controle especial: uma análise dos erros de medicação e indicadores de prescrição. Rev Eletrônica de Farmácia. 2016;13(1):45-54. mesmo estudo, quando avaliadas as prescrições feitas no setor privado, se observa uma prevalência de $18 \%$, mostrando uma variação significativa na frequência de dispensação deste medicamento em diferentes locais. Esses dados vão de encontro com o observado no presente estudo.

Com isso, ressalta-se que o uso indiscriminado de medicamentos se tornou um problema de saúde pública e, estratégias vêm sendo elaboradas com o objetivo de minimizar os riscos à saúde da população devido ao uso incorreto. Nesse sentido, o farmacêutico ocupa um papel fundamental na execução dessas estratégias e de ações que visem a orientação em relação ao uso de medicamentos controlados.

\section{CONCLUSÃO}

Com base nos resultados, observa-se que existe uma elevada taxa de dispensação de medicamentos controlados no município, levando em consideração a população atendida. Devido a ocorrência de vários efeitos adversos citados na literatura e, muitas vezes, dificuldades de adesão à terapia medicamentosa, seriam necessários mais critérios na prescrição desses medicamentos. Além disso, a atuação do profissional farmacêutico na dispensação desses medicamentos por meio da atenção farmacêutica é de extrema importância para fornecer aos pacientes as orientações necessárias à sua correta utilização.

2. BRASIL. Portaria $n^{\circ} 344$, de 12 de maio de 1998. Aprova o Regulamento Técnico sobre substâncias e medicamentos sujeitos a controle especial. Diário Oficial da União, 1998. 
3. Souza M F. Uso de psicotrópicos no Brasil: uma revisão da literatura. Journal of Biology \& Pharmacy and Agricultural Management. 2018;12(4):6-10.

4. Silva DP, Portugal FB, Júnior GMG. Análise da conformidade das prescrições medicamentosas dos setores públicos e privados. Rev Bras de Pesquisa em Saúde. 2017;19(1):78-84.

5. Prado MAMB, Francisco PMSB, Barros MBA. Uso de medicamentos psicotrópicos em adultos e idosos residentes em Campinas, São Paulo: um estudo transversal de base populacional. Epidemiologia e Serviços de Saúde. $2017 ; 26(4): 747-758$

6. IBGE. Instituto Brasileiro de Geografia e Estatística. Sinopse do Senso demográfico de 2010.

7. BRASIL, Ministério da Saúde. Secretaria de saúde do município de Boa Vista do Incra. Banco de dados de dispensação de medicamentos. Boa Vista do Incra, 2019.

8. Leite SN, Bernardo NLMC, Álvares J, Júnior AAG, Costa EA, Acurcio FA, et al. Serviço de dispensação de medicamentos na atenção básica no SUS. Rev de Saúde Pública. 2017; 51(suppl 2):1s-10s.

9. BRASIL. Lei $n^{\circ} 13.021$, de 8 de agosto de 2014. Dispõe sobre o exercício e a fiscalização das atividades farmacêuticas. Diário Oficial da União, 2014.

10. BRASIL. Ministério da Saúde. Resolução n 338 de 06 de maio de 2004. Aprova a Política Nacional de Assistência Farmacêutica. Diario Oficial Uniao. Brasília, p. 52, 2004.

11. BRASIL. Ministério da Saúde. Agência Nacional de Vigilância Sanitária (ANVISA). Resolução ${ }^{\circ} 22$ de 29 de abril de 2014. Dispõe sobre o Sistema Nacional de Gerenciamento de Produtos Controlados-SNGPC. Brasília, 2014.

12. IBGE, Instituto Brasileiro de Geografia e Estatística. Pesquisa nacional por amostra de domicílios contínua-PNAD contínua. Divulgação anual. 2018.

13. Santin I, Roman-Júnior WAR. Avaliação da dispensação de medicamentos pelo Sistema Único de Saúde no município de Vargem Bonita - Santa Catarina. Rev Bras de Farmácia. 2012; 93(2):161-166.

14. Alves-Júnior EBA, Bezerra ER, Diniz AFA, Alves LP, Nobrega RO, Felicio IM, et al. Utilização de benzodiazepínicos por usuários do sistema único de saúde. J Biology Pharmacy and Agricultural Management. 2017;13(3):17-21

15. Bittencourt SC, Caponi S, Maluf S. Medicamentos antidepressivos: inserção na prática biomédica (1941 a 2006) a partir da divulgação em um livro-texto de farmacologia. Mana. 2013; 19(2):219-247.

16. Ait-Daoud N, Hamby AS, Sharma S, Blevins D. A review of alprazolam use, misuse, and withdrawal. J addiction Medicine. 2018; 12(1):4-10.

17. Piper BJ, Suarez MJ, Piserchio JP, Shah DT, Simoyan $\mathrm{OM}, \mathrm{McC}$ all $\mathrm{KL}$, et al. Illicit and prescription drug misuse as reported to the Maine Diversion Alert Program. Forensic Science International, 2018; 285:65-71.
18. Votaw VR, Geyer R, Rieselbach MM, McHugh RK. The epidemiology of benzodiazepine misuse: A systematic review. Drug and alcohol dependence. 2019; 1(200):95-114

19. Vijayakumar S. Alprazolam. J Mental Health Nursing. 2017; $5(1): 46-49$

20. Silva ERB, Bandeira VAC, Oliveira KR. Avaliação das prescrições dispensadas em uma farmácia comunitária no município de São Luiz Gonzaga-RS. Rev de Ciências Farmacêuticas Básica e Aplicada. 2012; 33(2):275-281.

21. Schult RF, Morris AJ, Picard L, Wiegand TJ. Citalopram overdose and severe serotonin syndrome in an intermediate metabolizing patient. Am J of Emergency Medicine. 2019; 37(10):1993.e5-1993.e6.

22. Aguiar CC, Castro TR, Carvalho AF, Vale OC, Sousa FC, Vasconcelos SM. Drogas antidepressivas. Acta Medica Portuguesa. 2011; 24(1):91- 98.

23. Costa M, Marins N. Hyponatremia associated with antidepressants: a review. J Bras de Psiquiatria. 2018; 67(1):52 58.

24. Schenkel M, Colet CF. Uso de antidepressivos em um município do Rio Grande do Sul. Arquivos de Ciências da Saúde da UNIPAR. 2016;20(1):33-42.

25. Mccarrell JL, Bailey TA, Duncan NA, Covington LP, Clifford $\mathrm{KM}$, Hall RG, et al. A review of citalopram dose restrictions in the treatment of neuropsychiatric disorders in older adults. Mental Health Clinician. 2019; 9(4): 280-286.

26. Camelo AEM, Dinelly CM, Oliveira MAS. Psicotrópicos: perfil de prescrições de benzodiazepínicos, antidepressivos e anorexígenos a partir de uma revisão sistemática. Rev Eletrônica de Farmácia. 2016;13(3):111-122.

27. Ferreira KV, Melo NI. DEPRESSÃO EM IDOSOS: o papel do profissional farmacêutico. Psicologia e Saúde em debate. 2018; 4(1):44-60.

28. Soares SB, Muniz SDB, Albuquerque FGF, Malaquias IS Leite FC. Avaliação de uso de antidepressivos em uma farmácia privada na cidade de Cajazeiras-PB. J Biology \& Pharmacy and Agricultural Management. 2019; 15(3):282294.

29. Gonçalves DP, Silva IV, Rangel LB, Rezende LC. Prescription of psychoactive drugs in patients attended by the SUS at Manhuaçu-MG (Brazil). Pharmacy Practice. 2011; 9(4):200-206.

30. Alves MMO, Oliveira CSP. Dispensação de Antidepressivos em Farmácias do Setor Público e Privado do Município de Tijucas do Sul-PR. Rev Uniandrade. 2015; 16(3):160-166.

\section{CORRESPONDÊNCIA}

Jéssica Martins Prediger

Rodovia Municipal Jacob Della Méa, km 5.6 - Parada Benito

Cruz Alta - Rio Grande do Sul - CEP 98005-972

E-mail: jessica.bvicenter@hotmail.com 